\title{
Joint latent class analysis for longitudinal data: an application on adolescent emotional well-being
}

\author{
Eun Ah Kim ${ }^{a}$, Hwan Chung ${ }^{a}$, Saebom Jeon ${ }^{1, b}$ \\ ${ }^{a}$ Department of Statistics, Korea University, Korea; \\ ${ }^{b}$ Department of Marketing Information Consulting, Mokwon University, Korea
}

\begin{abstract}
This study proposes generalized models of joint latent class analysis (JLCA) for longitudinal data in two approaches, a JLCA with latent profile (JLCPA) and a JLCA with latent transition (JLTA). Our models reflect crosssectional as well as longitudinal dependence among multiple latent classes and track multiple class-sequences over time. For the identifiability and meaningful inference, EM algorithm produces maximum-likelihood estimates under local independence assumptions. As an empirical analysis, we apply our models to track the joint patterns of adolescent depression and anxiety among US adolescents and show that both JLCPA and JLTA identify three adolescent emotional well-being subgroups. In addition, JLCPA classifies two representative profiles for these emotional well-being subgroups across time, and these profiles have different tendencies according to the parent-adolescent-relationship subgroups.
\end{abstract}

Keywords: joint latent class profile analysis, joint latent transition analysis, EM algorithm, adolescent depression

\section{Introduction}

According to the 2017 National Survey on Drug Use and Health (NSDUH), about 13.3\% of adolescents aged 12 to 17 in the United States have experienced a depressive episode (Center for Behavioral Health Statistics and Quality, 2018). Adolescent depression is a major concern for youth development that relates to physical development and cognitive development as well as success in school, work, and society. Several researchers have suggested that depression in adolescence is strongly linked with anxiety, but knowledge of the mechanisms underlying this link remains inconclusive (Garber and Weersing, 2010; Horn and Wuyek, 2010; Schleider et al., 2014). The sequentially comorbid relationship between anxiety and depression likely involves a wide variety of cognitive, social, environmental, and biological factors (Schleider et al., 2014). For example, mutual trust and harmonious relationships between parents and adolescents have been found to have a positive impact on youth health (Gutman et al., 2005; White and Roosa, 2012).

In many studies, adolescent mental health have been inferred from responses to several related questionnaires rather than directly measured as a single scale, but it has been rarely considered as a latent variable. Some research has written recently used latent class analysis (LCA) to categorically classify subpopulations for depressive and anxiety symptoms (van Lang et al., 2006; Kreuter et al., 2008). LCA is a person-centered approach that can capture heterogeneity within and between subgroups, unlike factor analysis, which is a variable-centered approach (Nurius and Macy, 2008). There

\footnotetext{
${ }^{1}$ Corresponding author: Department of Marketing Information Consulting, Mokwon University, Doanbuk-ro 88, Seogu,

Daejeon 35349, Korea. E-mail: alwaysns@mokwon.ac.kr
}

Published 31 March 2020 / journal homepage: http://csam.or.kr

(c) 2020 The Korean Statistical Society, and Korean International Statistical Society. All rights reserved. 


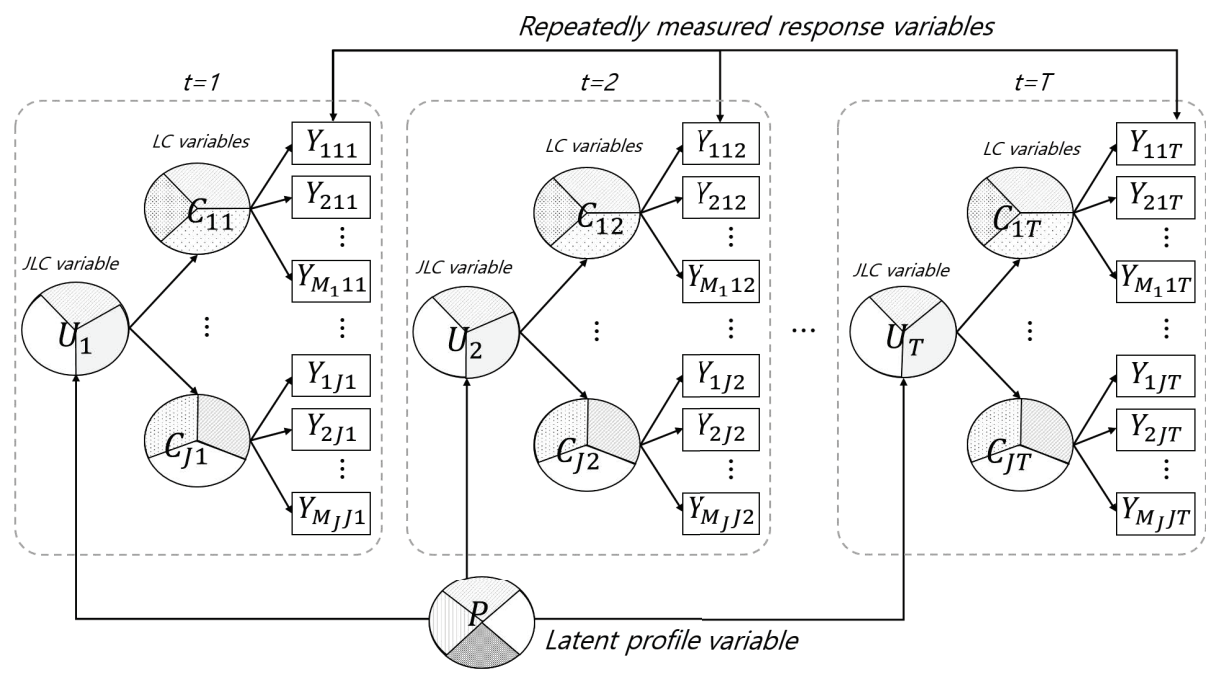

Figure 1: Model concept of joint latent class profile analysis.

are LCA variations for longitudinal data including latent transition analysis (LTA) and latent class profile analysis (LCPA) (Chung et al., 2008, 2011; Collins and Lanza, 2010). LTA and LCPA use a set of repeatedly measured manifest items to track latent transition or the path of a latent classes; however, and these are not suitable for two or more latent class variables. Joint LCA (JLCA) is another LCA variation for cross-sectional data that allows several latent variables simultaneously under multiple manifest item sets of different attributes (Jeon et al., 2017). For example, JLCA can consider multi-dimensional information of youth behavioral risk (joint latent variable) based on responses to substance use, violence, depression and suicide (each 4 latent variable). JLCA allows us to identify the association of different latent variables; however, it is limited to cross-sectional dependencies.

In this regard, this paper develops generalized latent class models that reflect cross-sectional dependence as well as longitudinal dependence between latent variables. In order to explain the dynamic changes or developments of multiple latent variables over time, we propose two types of new LC models of JLCA combined with the LTA concept and JLCA combined with the LCPA concept. For the parameter estimation, maximum likelihood estimation using an EM algorithm are used. As an empirical analysis, we apply our models to examine stage-sequential patterns of adolescent emotional well-being considering parent-adolescent relationships, using the data from Angel et al. (2009).

The rest of the paper is organized as follows. In Section 2, the model concepts and descriptions of the proposed JLCA with latent transition (JLTA) and JLCA with latent profile (JLCPA) are presented. Section 3 describes estimation methods for the parameters and model diagnosis. In Section 4, empirical analysis results are represented, and Section 5 concludes the paper.

\section{Joint latent class analysis for longitudinal data}

JLCA explores the joint action or association of multiple latent variables only for a static phenomena; therefore, we develop two JLC variation models by incorporating the JLCA with two typical latent class (LC) models for longitudinal data of LCPA and LTA. These two models allow the investigation of changes in multiple latent class variables over time that reflect longitudinal dependence. 


\subsection{Joint latent class profile analysis}

Joint LCPA (JLCPA) is an extended LCPA where multiple latent variables are allowed to be associated and their latent paths can be tracked using sets of repeatedly measured manifest items. JLCPA also postulates a joint latent variable composed of several latent variables at each measurement time point. Figure 1 illustrats JLCA at each time $t$ and its profile identified by sequences of joint latent class memberships across time.

Suppose that there are $J$ latent class variables $C_{1 t}, \ldots, C_{J t}$ and the variable $C_{j t}$ consists of $K_{j}$ categories at time $t$. For a given $t$, the $\mathrm{LC}$ variable $C_{j t}$ was identified from the $j^{\text {th }}$ item set $\mathbf{Y}_{j t}=$ $\left(Y_{1 j t}, \ldots, Y_{M_{j} j t}\right)^{\prime}$ and the manifest item $Y_{m_{j} j t}$ ranges from 1 to $r_{m_{j}}$ for $j=1, \ldots, J$. Let a joint class variable $U_{t}$ at time $t$ has $S$ nominal categories to describe the common joint patterns of $J$ latent class memberships, and a latent profile variable $P$ has $L$ nominal profiles to describe the representative joint class sequences over time. Let $\mathbf{y}_{i j t}=\left(y_{i 1 j t}, \ldots, y_{i M_{j} j t}\right)^{\prime}$ be the $i^{t h}$ individual's responses to a set of $M_{j}$ manifest items measuring a $j^{\text {th }}$ latent feature at time $t$, then the complete-data likelihood of the $i^{\text {th }}$ individual over time can be presented as

$$
\begin{aligned}
L_{(\mathrm{JLCPA}) i}^{*} & =P\left(P=l, \mathbf{U}=\mathbf{s}, \mathbf{C}=\mathbf{c}, \mathbf{Y}_{i}=\mathbf{y}_{i}\right) \\
& =P(P=l) P(\mathbf{U}=\mathbf{s} \mid P=l) P(\mathbf{C}=\mathbf{c} \mid \mathbf{U}=\mathbf{s}) P\left(\mathbf{Y}_{i}=\mathbf{y}_{i} \mid \mathbf{C}=\mathbf{c}\right) \\
& =\delta_{l} \prod_{t=1}^{T}\left\{\gamma_{s_{t} \mid l}^{(t)} \prod_{j=1}^{J}\left[\eta_{c_{j} \mid s_{t}}^{(j, t)} \prod_{m_{j}=1}^{M_{j}} \prod_{k=1}^{r_{m_{j}}} \rho_{m_{j} k j \mid c_{j}}^{(t)} I\left(y_{i m_{j} j t}=k\right)\right]\right\},
\end{aligned}
$$

where $I\left(y_{i m_{j} j t}=k\right)$ is an indicator function that is equal to 1 when $y_{i m_{j} j t}=k$ and 0 otherwise. The primary measurement parameter $\rho_{m_{j} k j \mid c_{j}}^{(t)}$ denotes the probability that an individual responds $k$ to the $m_{j}^{\text {th }}$ item of the $j^{\text {th }}$ latent variable, for a given latent class $c_{j t}$ at time $t$. The secondary measurement parameter $\eta_{c_{j} \mid s_{t}}^{(j, t)}$ represents the conditional probability that an individual belongs to a class $c_{j t}$ of $j^{\text {th }}$ latent variable at time $t$, for a given joint latent class $s_{t}$ at time $t$. The tertiary measurement parameter $\gamma_{s_{t} \mid l}^{(t)}$ is the conditional probability that an individual belongs to a joint class $s$ at time $t$, for a given profile $l$. Finally, $\delta_{l}$ represents the probability that an individual belongs to a profile $l$.

The likelihood of JLCPA is based on the following assumptions: (a) both the joint class membership and the joint class profile membership are related to the manifest items only through the class membership; (b) the manifest items are conditionally independent given a class membership; (c) given a joint class membership, each class membership is unrelated; and (d) the joint class membership is unrelated within a joint class profile. Assumptions (b), (c), and (d) indicate local independence which allows the inference of the unobserved classes, joint class, and joint class profile.

The observed-data likelihood, the likelihood of manifest items, can be derived by marginalizing the complete-data likelihood over all the considered latent variables $\mathbf{C}, \mathbf{U}, P$ :

$$
L_{(\mathrm{JLCPA}) i}=P\left(\mathbf{Y}_{i}=\mathbf{y}_{i}\right)=\sum_{l=1}^{L} \sum_{s_{1}=1}^{S} \ldots \sum_{s_{T}=1}^{S} \sum_{c_{1}=1}^{K_{1}} \cdots \sum_{c_{J}=1}^{K_{J}} L_{(\mathrm{JLCPA}) i}^{*}
$$

\subsection{Joint latent transition analysis}

The second model is a joint LTA (JLTA) that identifies the marginal joint class prevalence at the initial time and latent transitions over two consecutive time points, instead of a latent profile or common pathways of joint class. We assume that the sequence of $U_{i t}$ constitutes a first-order Markov chain for 
$t=1, \ldots, T$. Let $\tau_{s_{t} \mid s_{t-1}}$ be a $S \times S$ transition matrix representing the probability that an individual belongs to a joint class $s_{t}$ at time $t$ given $s_{t-1}$ at time $t-1$, for $s_{t-1}=1, \ldots, S$ and $s_{t}=1, \ldots, S$. The complete-data likelihood of JLTA can be derived by substituting the prevalence of joint class profile $\delta_{l}$ and the tertiary measurement parameter $\gamma_{s_{t} \mid l}^{(t)}$ into the prevalence of joint latent class at initial time $\delta_{s_{1}}$ and the transition probability $\tau_{s_{t} \mid s_{t-1}}^{(t)}$. Then, the complete-data likelihood is

$$
\begin{aligned}
L_{(\mathrm{JLTA}) i}^{*} & =P\left(\mathbf{U}=\mathbf{s}, \mathbf{C}=\mathbf{c}, \mathbf{Y}_{i}=\mathbf{y}_{i}\right) \\
& =\delta_{s_{1}} \prod_{t=2}^{T} \tau_{s_{t} \mid s_{t-1}}^{(t)} \prod_{t=1}^{T} \prod_{j=1}^{J}\left[\eta_{c_{j} \mid s_{t}}^{(j, t)} \prod_{m_{j}=1}^{M_{j}} \prod_{k=1}^{r_{m_{j}}} \rho_{m_{j} k j \mid c_{j}}^{(t)} I\left(y_{i m_{j} j t}=k\right)\right],
\end{aligned}
$$

where $I\left(y_{i m_{j} j t}=k\right)$ is an indicator function that is equal to 1 when $y_{i m_{j} j t}=k$ and 0 otherwise. The primary measurement parameter $\rho_{m_{j} k j c_{j}}^{(t)}$ represents the probability that an individual responds $k$ to the $m_{j}^{\text {th }}$ item of the $j^{\text {th }}$ latent variable, for a given latent class $c_{j t}$ at time $t$, and the secondary measurement parameter $\eta_{c_{j} \mid s_{t}}^{(j, t)}$ is the conditional probability that an individual belongs to a class $c_{j t}$ of the $j^{\text {th }}$ latent variable at time $t$ for a given joint latent class $s_{t}$ at time $t$. Finally, $\delta_{s_{1}}$ is the prevalence of joint latent class at initial time, and $\tau_{s_{t} \mid s_{t-1}}^{(t)}$ is the transition probability.

JLTA also requires the local independence assumptions for meaningful inferences about all latent variables. The observed-data likelihood can be obtained as

$$
L_{(\mathrm{JLTA}) i}=P\left(\mathbf{Y}_{i}=\mathbf{y}_{i}\right)=\sum_{s_{1}=1}^{S} \cdots \sum_{s_{T}=1}^{S} \sum_{c_{1}=1}^{K_{1}} \cdots \sum_{c_{J}=1}^{K_{J}} L_{(\mathrm{JLTA}) i}^{*}
$$

\section{Parameter estimation and model diagnosis}

Expectation-Maximization (EM) algorithm is the most widely used estimation method for LC-type models (Mooijaart and Van der Heijden, 1992; Jeon et al., 2017; Lee and Chung, 2017). EM provides ML estimates even for missing data under missing at random assumption, and can yield stable estimates regardless of boundary solutions. The EM algorithm consists of the E-step and M-step, and once given with appropriate initial value, the two steps are repeated until the estimates converge to fixed points.

\subsection{Estimation of JLCPA}

E-step calculates the expectation of the log-complete data likelihood under the current estimates of the parameters. The expectation is expressed as a function of posterior probabilities that individual $i$ belongs to profile $l$, the joint class sequence $\mathbf{s}=\left(s_{1}, \ldots, s_{T}\right)$ and the class $\mathbf{c}=\left(\mathbf{c}_{1}, \ldots, \mathbf{c}_{T}\right)$, given a set of manifest item responses $\mathbf{y}_{i}=\left(\mathbf{y}_{i 1}, \ldots, \mathbf{y}_{i T}\right)$, and calculated using the tentative parameter estimates from the previous iteration. The posterior probabilities can be obtained by $\theta_{i(l, \mathbf{s}, \mathbf{c})}=P(P=l, \mathbf{U}=$ $\left.\mathbf{s}, \mathbf{C}=\mathbf{c} \mid \mathbf{Y}_{i}=\mathbf{y}_{i}\right)=L_{(\mathrm{JLCPA}) i}^{*} / L_{(\mathrm{JLCPA}) i}$. As the complete-data likelihood $L_{(\mathrm{JLCPA}) i}^{*}$ in equation (2.1) can be expressed with indicator functions as

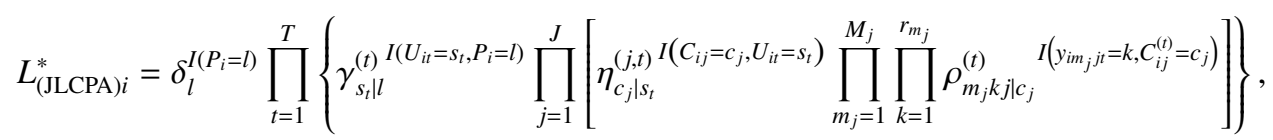


and the expectation of log-complete-data likelihood can be derived as follows.

$$
\begin{aligned}
E\left(\sum_{i=1}^{n} \log L_{(\mathrm{JLCPA}) i}^{*}\right)= & \sum_{i=1}^{n} \sum_{l=1}^{L} \theta_{i(l)} \log \delta_{l} \\
& +\sum_{i=1}^{n} \sum_{t=1}^{T} \sum_{s_{t}=1}^{S} \sum_{l=1}^{L} \theta_{i\left(l, s_{t}\right)}^{(t)} \log \gamma_{s_{t} l l}^{(t)} \\
& +\sum_{i=1}^{n} \sum_{t=1}^{T} \sum_{j=1}^{J} \sum_{c_{j}=1}^{C_{j}} \sum_{s_{t}=1}^{S} \theta_{i\left(s_{t}, c_{j}\right)}^{(t)} \log \eta_{c_{j} \mid s_{t}}^{(j, t)} \\
& +\sum_{i=1}^{n} \sum_{t=1}^{T} \sum_{j=1}^{J}\left\{\sum_{c_{j}=1}^{C_{j}} \theta_{i\left(c_{j}\right)}^{(t)}\left[\sum_{m_{j}=1}^{M_{j}} \sum_{k=1}^{r_{m_{j}}} I\left(y_{i m_{j} j}^{(t)}=k\right) \log \rho_{m_{j} k j c_{j}}^{(t)}\right]\right\} .
\end{aligned}
$$

where $\theta_{i(l)}=\sum_{s_{t}} \theta_{i\left(l, s_{t}\right)}^{(t)}, \theta_{i\left(l, s_{t}\right)}^{(t)}=\sum_{c_{1}} \cdots \sum_{c_{J}} \theta_{i\left(l, s_{t}, \mathbf{c}\right)}^{(t)}, \theta_{i\left(s_{t}, c_{j}\right)}^{(t)}=\sum_{l} \sum_{c_{1}} \cdots \sum_{c_{j-1}} \sum_{c_{j+1}} \cdots \sum_{c_{J}} \theta_{i\left(l, s_{t}, \mathbf{c},\right.}^{(t)}$, and $\theta_{i\left(c_{j}\right)}^{(t)}=\sum_{s_{t}} \theta_{i\left(s_{t}, c_{j}\right)}^{(t)}$. Then, ML estimates for JLCPA can be obtained by taking partial derivatives and solving normal equations. The model parameters can therefor be derived by

$$
\hat{\delta}_{l}=\frac{\sum_{i} \theta_{i l}}{n}, \quad \hat{\gamma}_{s_{l} \mid l}^{(t)}=\frac{\sum_{i} \theta_{i\left(l, s_{t}\right)}^{(t)}}{\sum_{i} \theta_{i l}}, \hat{\eta}_{c_{j} \mid s_{t}}^{(j, t)}=\frac{\sum_{i} \theta_{i\left(s_{t}, c_{j}\right)}^{(t)}}{\sum_{i} \theta_{i\left(s_{t}\right)}^{(t)}}, \quad \text { and } \quad \hat{\rho}_{m_{j} k j \mid c_{j}}^{(t)}=\frac{\sum_{i} \theta_{i\left(c_{j}\right)}^{(t)} \times I\left(y_{i m_{j} j}^{(t)}=k\right)}{\sum_{i} \theta_{i\left(c_{j}\right)}^{(t)}} .
$$

For the invariant $\eta$ or $\rho$ over time for practical interpretation due to the consistent meaning of latent classes across time, the parameter estimators become

$$
\hat{\eta}_{c_{j} \mid s_{t}}^{(j)}=\frac{\sum_{t=1}^{T} \sum_{i} \theta_{i\left(s_{t}, c_{j}\right)}^{(t)}}{\sum_{t=1}^{T} \sum_{i} \theta_{i\left(s_{t}\right)}^{(t)}} \quad \text { and } \quad \hat{\rho}_{m_{j} k j c_{j}}=\frac{\sum_{t=1}^{T}\left\{\sum_{i} \theta_{i\left(c_{j}\right)}^{(t)} \times I\left(y_{i m_{j} j}^{(t)}=k\right)\right\}}{\sum_{t=1}^{T} \sum_{i} \theta_{i\left(c_{j}\right)}^{(t)}} .
$$

\subsection{Estimation of JLTA}

E-step computes the conditional probability that the $i^{\text {th }}$ individual belongs to stage sequence $\mathbf{s}=$ $\left(s_{1}, \ldots, s_{T}\right)$ and the class $\mathbf{c}=\left(\mathbf{c}_{\mathbf{1}}, \ldots, \mathbf{c}_{\mathbf{T}}\right)$ given $\mathbf{y}_{i}$. The posterior probabilities can be obtained by $\theta_{i}(\mathbf{s}, \mathbf{c})=P\left(\mathbf{U}=\mathbf{s}, \mathbf{C}=\mathbf{c} \mid \mathbf{Y}_{i}=\mathbf{y}_{i}\right)=L_{(\mathrm{JLTA}) i}^{*} / L_{(\mathrm{JLTA}) i}$. Using the ordinary indicator functions, the complete-data likelihood derived in (2.3) can be expressed as follows.

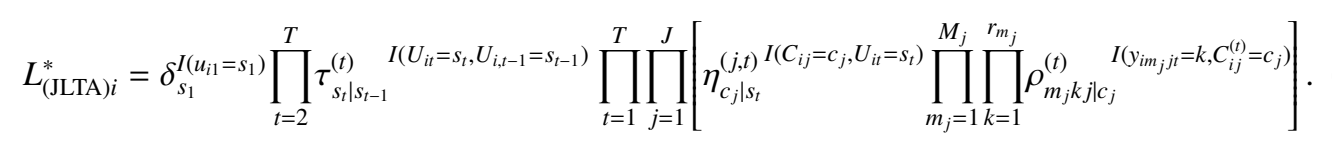

Using the likelihood given in (3.5), the expectation of the log-complete data likelihood can be obtained in terms of $\theta$,

$$
\begin{aligned}
E\left(\sum_{i=1}^{n} \log L_{(\mathrm{JLTA}) i}^{*}\right)= & \sum_{i=1}^{n} \sum_{s_{1}=1}^{S} \theta_{i\left(s_{1}\right)} \log \left(\delta_{s_{1}}\right) \\
& +\sum_{i=1}^{n} \sum_{t=2}^{T} \sum_{s_{t}=1}^{S} \sum_{s_{t-1}=1}^{S} \theta_{i\left(s_{t}, s_{t-1}\right)}^{(t)} \log \tau_{s_{t} \mid s_{t-1}}^{(t)}
\end{aligned}
$$




$$
\begin{aligned}
& +\sum_{i=1}^{n} \sum_{t=1}^{T} \sum_{j=1}^{J} \sum_{c_{j}=1}^{C_{j}} \sum_{s_{t}=1}^{S} \theta_{i\left(s_{t}, c_{j}\right)}^{(t)} \log \eta_{c_{j} \mid s_{t}}^{(j, t)} \\
& +\sum_{i=1}^{n} \sum_{t=1}^{T} \sum_{j=1}^{J}\left\{\sum_{c_{j}=1}^{C_{j}} \theta_{i\left(c_{j}\right)}^{(t)}\left[\sum_{m_{j}=1}^{M_{j}} \sum_{k=1}^{r_{m_{j}}} I\left(y_{i m_{j} j}^{(t)}=k\right) \log \rho_{m_{j} k j \mid c_{j}}^{(t)}\right]\right\} .
\end{aligned}
$$

The M-step focuses on the maximization of the expected complete-data likelihood of JLCA model with latent transition with respect to the model parameters. The resulting parameter estimators are

$$
\hat{\delta}_{s_{1}}=\frac{\sum_{i} \theta_{i\left(s_{1}\right)}}{n}, \quad \hat{\tau}_{s_{t} \mid s_{t-1}}^{(t)}=\frac{\sum_{i} \theta_{i\left(s_{t}, s_{t-1}\right)}^{(t)}}{\sum_{i} \theta_{s_{t-1}}^{(t)}}, \hat{\eta}_{c_{j} \mid s_{t}}^{(j, t)}=\frac{\sum_{i} \theta_{i\left(s_{t}, c_{j}\right)}^{(t)}}{\sum_{i} \theta_{i\left(s_{t}\right)}^{(t)}}, \quad \text { and } \quad \hat{\rho}_{m_{j} k j \mid c_{j}}^{(t)}=\frac{\sum_{i} \theta_{i\left(c_{j}\right)}^{(t)} \times I\left(y_{i m_{j} j}^{(t)}=k\right)}{\sum_{i} \theta_{i\left(c_{j}\right)}^{(t)}} .
$$

The parameters of $\eta$ and $\rho$ become (3.4) under the invariant assumption over time, in the same way in JLCPA. For $\tau$, it may be of interest to consider the invariance assumption of the transition probability matrix across time to simplify the model when $T \geq 3$. The null hypothesis of equal transition probabilities over time can be tested by comparing the model fit statistics of two models. The transition probability estimator under invariance assumption becomes

$$
\hat{\tau}_{s_{t} \mid s_{t-1}}=\frac{\sum_{t=2}^{T} \sum_{i} \theta_{i\left(s_{t}, s_{t-1}\right)}^{(t)}}{\sum_{t=2}^{T} \sum_{i} \theta_{s_{t-1}}^{(t)}}
$$

\subsection{Model diagnosis}

It is important to assess the model with objective fit measures to understand whether the fitted model is appropriate to the data. LC model which properly reflects the underlying latent structure of the data enables us draw valid statistical inferences. Pearson $\chi^{2}$ and LRT statistics generally do not hold for latent class model, and bootstrap $p$-value obtained from the empirical distribution can be considered as criterion for the absolute performance of the model (Chung et al., 2011). For the calculation of bootstrap $p$-value, we perform parametric bootstrap procedure by generating 100 bootstrap samples of $G^{2}$ statistic, and calculate the proportion of the bootstrapped $G^{2}$ s that are larger than observed $G^{2}$. The model whose bootstrap $p$-value is larger than the significance level is considered as a proper model for the data. Next, we can compare the performance of several plausible models and select the best one. The relative model fit statistics such as Akaike information criterion (AIC) and Bayesian information criterion (BIC) can be considered to determine which LC model is better for explaining a data set. AIC tends to prefer the complex model with a lot of parameters while BIC usually indicates that a comparatively simple model is the optimal model. The model with $p$-value to be larger than 0.05 , and the model with smaller AIC (or BIC) is relatively preferred, but the interpretability of the latent classes also should be considered in order to reflect the underlying latent structure of the data properly.

\section{Application to adolescents emotional well-being}

\subsection{Data description}

The study of Angel et al. (2009) analyzed a longitudinal survey data to assess the well-being of lowincome children and families in Boston, Chicago, and San Antonio. Each household had a family income below $200 \%$ of the federal poverty line, a female caregiver, and a child (aged 0 to 4 years) or 
Table 1: Selection of the number of latent class for each latent component at wave 1

\begin{tabular}{ccccc}
\hline \hline Latent variable & Number of classes & AIC & BIC & Bootstrap $p$-value \\
\hline \multirow{3}{*}{ Depression } & 2 & 4822.1 & 4883.6 & 0.00 \\
& 3 & 4807.0 & 4901.6 & 0.05 \\
& 4 & 4799.6 & 4927.4 & 0.39 \\
& 5 & 4801.6 & 4962.5 & 0.54 \\
\hline \multirow{3}{*}{ Anxiety } & 2 & 4585.0 & 4646.5 & 0.00 \\
& 3 & 4524.9 & 4619.5 & 0.71 \\
& 4 & 4527.2 & 4654.9 & 0.65 \\
& 5 & 4536.3 & 4697.2 & 0.48 \\
\hline \hline
\end{tabular}

$\mathrm{AIC}=$ Akaike information criterion; $\mathrm{BIC}=$ Bayesian information criterion.

adolescent (aged 10 to 14 years) at the time of the first interview. This study focuses on the $N=839$ sample households with adolescent, and their responses on the manifest items related to the adolescent emotional well-being and parent-adolescent relationship over three waves in 1999, 2001, and 2005.

For the study, we used 6 manifest items on the adolescent depression, 6 items measuring adolescent anxiety. Questionnaires on depression behaviors are "do you...?" (a) often feel no interest in things; (b) often feel lonely; (c) often feel blue; (d) often feel worthless; (e) often feel hopeless about future; (f) sometimes think about suicide. Items on anxiety behaviors are whether do you (a) often feel nervous; (b) often feel tense; (c) often feel scared suddenly; (d) often feel panic; (e) often feel restless; (f) often feel fearful. For the interpretational simplicity, all the polychotomous items were changed into dichotomous indicating true or not.

\subsection{Model selection}

JLCPA and JLTA have several latent variables and complicated structure over time which can cause identifiability problem. Prior to the construction of JLCPA (or JLTA) model, it is required to specify the appropriate latent structure of each latent variable. The joint latent structure of the multiple latent variables can be determined, once the latent structure of the each latent variable is specified (Jeon et al., 2017). The stability of model was examined by using 100 different random starting values to prevent a local optimum problem. We adopt the information criteria (AIC, BIC), and bootstrap $p$-value with significance level $\alpha=0.05$ to assess the goodness-of-fit, and we also take into account the interpretability of each latent class model.

Table 1 presents AIC, BIC and bootstrap $p$-value estimated using the data at wave 1 with varying number of latent classes. The best class structure for Depression is determined as 3-class model by considering AIC, BIC, bootstrap p-value and the interpretability of latent class. Similarly, the best class structure of the Anxiety variable is determined as 3-class model. The optimal number of classes for each latent variable hold the same for the data at wave 2 and 3.

Figure 2 represents the $\rho$-probabilities of manifest items for the 3 -class depression groups (left) and 3-class anxiety groups (right) at each wave. For both depression and anxiety groups, normal groups are illustrated as dotted line, mild groups as dashed line, and severe groups as solid line. Although the $\rho$-probabilities show slight fluctuations, their ranks and meaning of latent classes are stable over time. Based on these item response probabilities, the primary measurement parameters were constrained as invariant over time.

Based on the pre-specified latent structures, the optimal number of joint latent class was selected. We called the identified joint latent class variable as Emotional well-being. Goodness-of-fit measures were calculated and presented in Table 2 for the various joint latent classes. Similar to the previous procedure, the best structure for the joint latent variable is the model with three joint classes, showing 


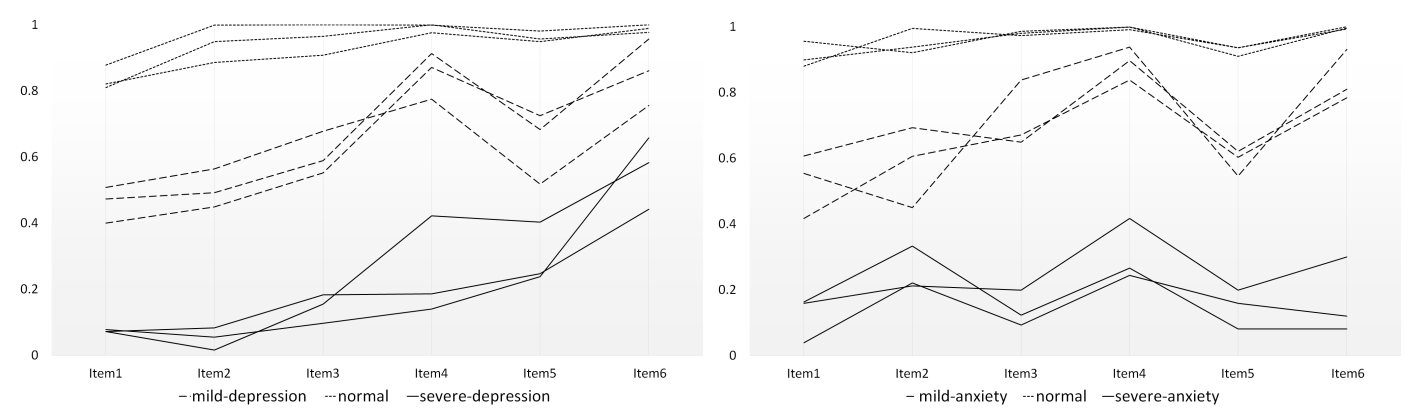

Figure 2: The estimated $\rho$-parameters of depression (left) and anxiety (right) at each wave.

Table 2: Selection of the number of joint latent class with different number of classes at wave 1

\begin{tabular}{ccccc}
\hline \hline Joint latent variable & Number of classes & AIC & BIC & $p$-value \\
\hline \multirow{3}{*}{ Emotional well-being } & 2 & 25963.75 & 26602.59 & 0.00 \\
& 3 & 25640.17 & 26350.00 & 1.00 \\
& 4 & 25678.25 & 26459.07 & 1.00 \\
\hline \hline
\end{tabular}

AIC = Akaike information criterion; BIC = Bayesian information criterion.

the smallest AIC, BIC and acceptable bootstrap p-value. At wave 2 and wave 3, the optimal number of joint latent classes also show the same.

Figure 3 represents the $\eta$-parameters of the 3-class depression groups (upper) and 3-class anxiety groups (lower) given three joint latent classes at each wave; each wave is illustrated as each dotted/dashed/solid line for each joint latent class. The $\eta$-parameters rarely fluctuate over time, and their meaning of latent classes given joint class is very stable over time, except for the mild-anxiety class given severe-symptoms, which can be due to its rareness. Therefore, the secondary measurement parameters $\eta$ are constrained to be invariant over time.

JLCPA has an extra number of latent component (latent profile) to be determined based on the pre-specified latent class and joint class structures. Table 3 presents the goodness-of-fit for JLTA and JLCPA with different number of profile, and the JLCPA model with 2-profile showed the smallest BIC and the model with 4-profile showed the smallest AIC. Considering model interpretability and law of parsimony, we decided to choose 2-profile latent model with 3-joint class, 3-class for Depression and 3-class for Anxiety as the best model (Figure 4). JLTA also appears to be a competitive model in terms of model fit; therefore, we infer from both JLCPA and JLTA.

\subsection{Parameter estimates}

We estimated the parameters of our interest under the selected model structure. Table 4 shows the estimated $\rho$-parameters of the latent variable for Depression and Anxiety. JLCPA and JLTA provide similar estimates for item-response probabilities. With regard to the Depression variable, individuals in the Normal class have small probabilities for all items, those in the Mild-depression class have higher probabilities than the Normal class, and those in the Severe-depression class have high probabilities of responding "Yes" to mild depressive symptoms and moderate probabilities for severe depressive symptoms. Similarly, with regard to the Anxiety variable, the item-response probabilities are small in the Normal class, medium in the Mild-anxiety class, and and high in the Severe-anxiety class showing the tendency of individuals belonging to the class.

Table 5 represents the probability of belonging to the class. From JLCPA results, the first joint 

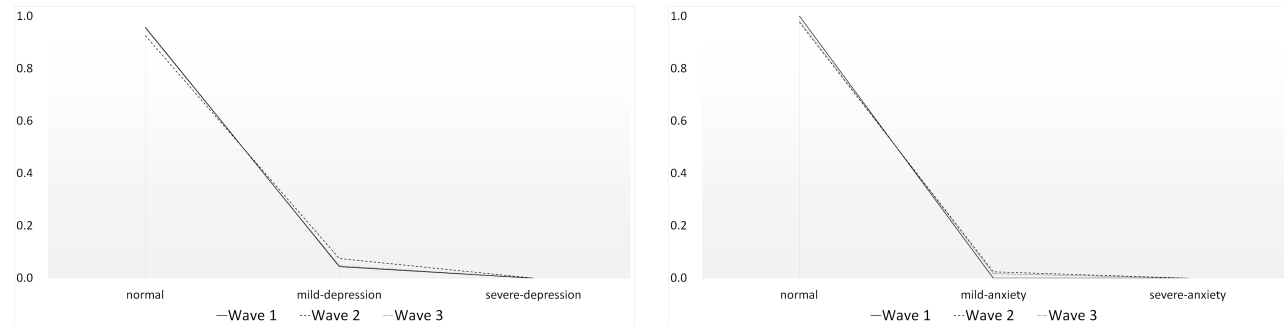

(a) Joint latent class 1

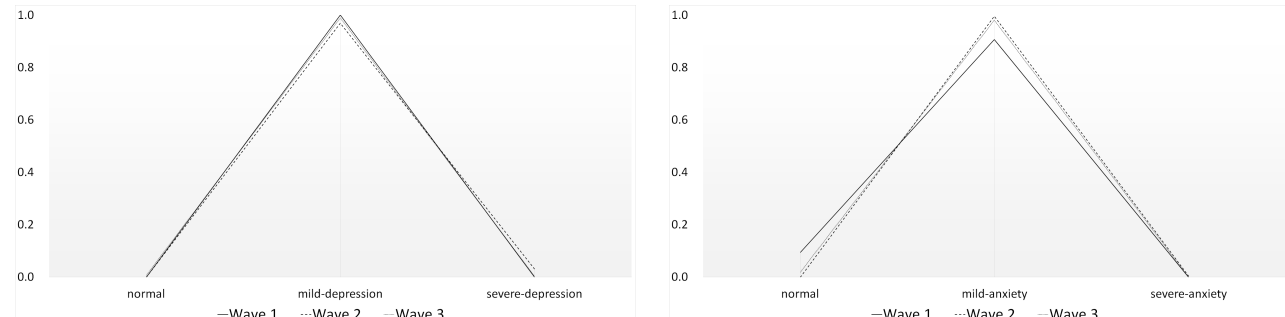

(b) Joint latent class 2

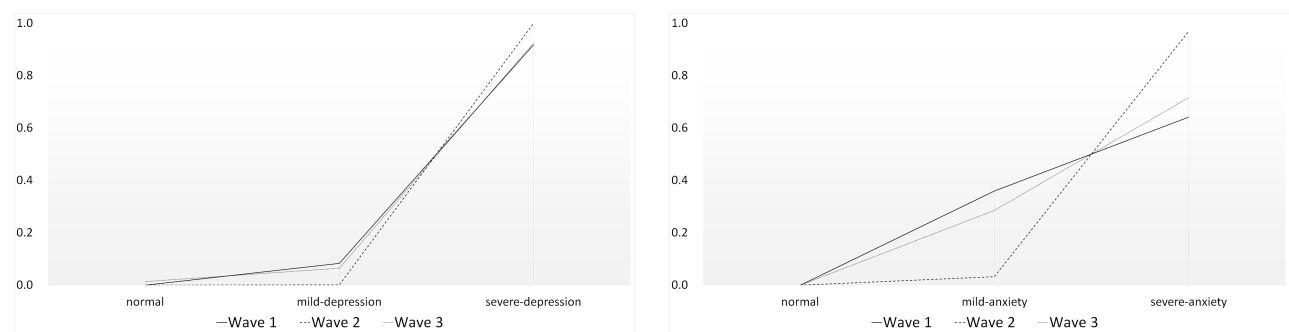

(c) Joint latent class 3

Figure 3: The estimated $\eta$-parameters of depression (left) and anxiety (right) at each wave.

Table 3: Diagnostic statistics for JLTA model and JLCPA models with the different number of profiles

\begin{tabular}{cccc}
\hline \hline Model & Number of profiles & AIC & BIC \\
\hline \multirow{3}{*}{ JLCPA } & 2 & 31898.03 & 32409.08 \\
& 3 & 31893.10 & 32494.99 \\
& 4 & 31805.02 & 32495.93 \\
\hline JLTA & N/A & 31879.14 & 32413.87 \\
\hline \hline
\end{tabular}

JLTA = joint latent transition analysis; JLCPA = joint latent class profile analysis; AIC = Akaike information criterion; $\mathrm{BIC}=$ Bayesian information criterion

class can be labeled as the Normal emotions because the probabilities of belonging to the class of Normal are 0.937 and 0.993 for the Depression and Anxiety. The second joint class can be named as Mild symptoms as the probability of belonging to the class of Mild-depression for the Depression and Mild-anxiety for the Anxiety are both over 0.9. The last joint class shows the high probability of belonging to the class of Severe-depression and Severe-anxiety, so it can be labeled as Severe symptoms. JLTA also shows similar results.

Based on these latent structure, the conditional probabilities of joint class membership for a particular class profile (the tertiary measurement parameters of JLCPA) identified the representative se- 


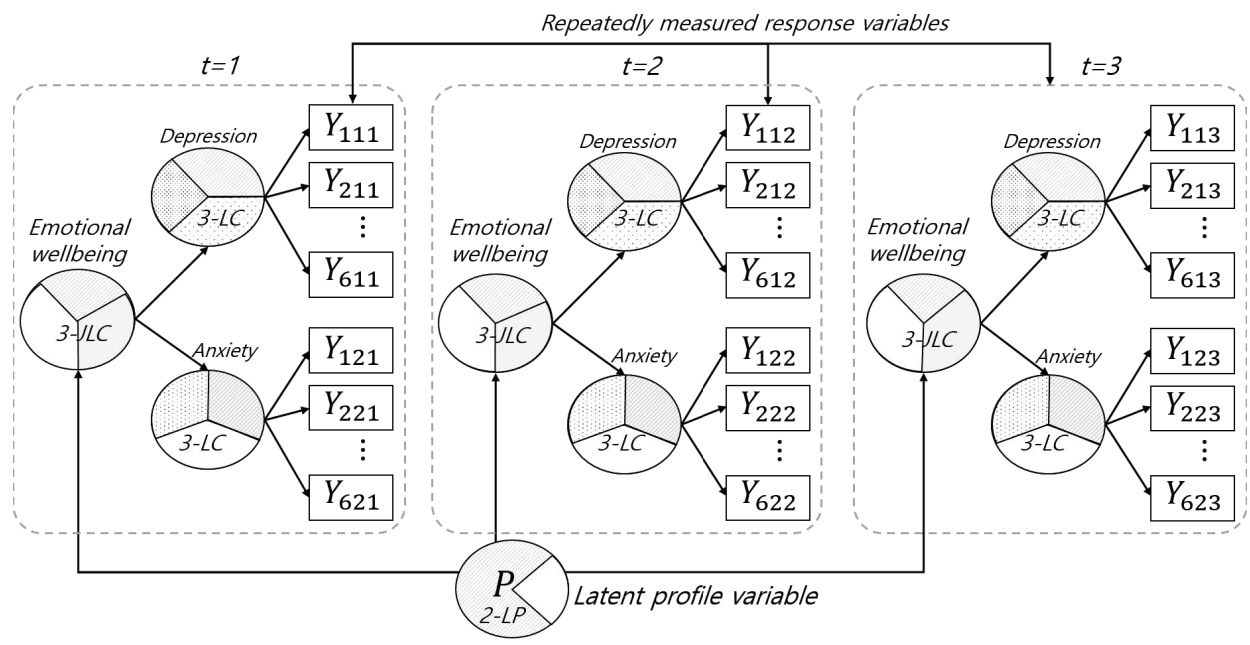

Figure 4: Model structure of the selected joint latent class profile analysis.

Table 4: The estimated $\rho$-parameters of item-response probabilities for each latent variable

\begin{tabular}{|c|c|c|c|c|c|c|}
\hline \multirow{3}{*}{ Manifest item } & \multicolumn{6}{|c|}{ Latent class for Depression } \\
\hline & \multicolumn{3}{|c|}{ JLCPA } & \multicolumn{3}{|c|}{ JLTA } \\
\hline & Normal & Mild-depression & Severe-depression & Normal & Mild-depression & Severe-depression \\
\hline Feeling no interest in things & 0.134 & 0.590 & 0.922 & 0.132 & 0.585 & 0.922 \\
\hline Feeling lonely & 0.061 & 0.508 & 0.953 & 0.059 & 0.501 & 0.947 \\
\hline Feeling blue & 0.045 & 0.412 & 0.861 & 0.043 & 0.410 & 0.859 \\
\hline Feeling worthless & 0.006 & 0.154 & 0.791 & 0.006 & 0.150 & 0.791 \\
\hline Feeling hopeless about future & 0.052 & 0.315 & 0.763 & 0.050 & 0.313 & 0.762 \\
\hline Sometimes think about suicide & 0.011 & 0.119 & 0.482 & 0.012 & 0.117 & 0.480 \\
\hline \multirow{3}{*}{ Manifest item } & \multicolumn{6}{|c|}{ Latent class for Anxiety } \\
\hline & \multicolumn{3}{|c|}{ JLCPA } & \multicolumn{3}{|c|}{ JLTA } \\
\hline & Normal & Mild-anxiety & Severe-anxiety & Normal & Mild-anxiety & Severe-anxiety \\
\hline Feeling nervous & 0.079 & 0.455 & 0.893 & 0.077 & 0.448 & 0.890 \\
\hline Feeling tense & 0.038 & 0.401 & 0.775 & 0.035 & 0.396 & 0.771 \\
\hline Feeling scared suddenly & 0.032 & 0.256 & 0.858 & 0.031 & 0.252 & 0.846 \\
\hline Feeling panic & 0.004 & 0.106 & 0.662 & 0.004 & 0.103 & 0.649 \\
\hline Feeling restless & 0.063 & 0.411 & 0.827 & 0.060 & 0.405 & 0.826 \\
\hline Feeling fearful & 0.010 & 0.147 & 0.812 & 0.010 & 0.140 & 0.807 \\
\hline
\end{tabular}

JLCPA $=$ joint latent class profile analysis; JLTA $=$ joint latent transition analysis.

quential patterns of Adolescent emotional well-being. In Table 6, the most common profile can be labeled as Deterioration and its prevalence is $71.6 \%$. Among individuals in 'Deterioration,' the probabilities of belonging to the joint class of Normal emotions are consistently decreased over time and those of belonging to other class of Mild symptoms are slightly increased over time (see the first two rows in Table 6). The model also identified other profiles of juveniles, some of whom tended to intensify their depressive and anxious symptoms over time and others who got better, moving towards Normal emotions or Severe symptoms from Mild symptoms, labeled as 'Polarization.'

Table 7 presents the estimates of marginal probabilities at wave 1 and transition probabilities from JLTA. At wave 1, the class membership of Normal emotions is 0.503 , that of Mild symptoms is 0.390 , and that of Severe symptoms is 0.107 . At wave 2, individuals among Normal emotions class at wave 
Table 5: The estimated $\eta$-parameters of belonging to a class for each given joint class

\begin{tabular}{|c|c|c|c|c|c|c|c|}
\hline \multirow{3}{*}{$\begin{array}{c}\text { Latent } \\
\text { variable }\end{array}$} & \multirow{3}{*}{ Class } & \multicolumn{6}{|c|}{ Joint class for Emotional well-being } \\
\hline & & \multicolumn{3}{|c|}{ JLCPA } & \multicolumn{3}{|c|}{ JLTA } \\
\hline & & $\begin{array}{c}\text { Normal } \\
\text { emotions }\end{array}$ & $\begin{array}{c}\text { Mild } \\
\text { symptoms }\end{array}$ & $\begin{array}{c}\text { Severe } \\
\text { symptoms }\end{array}$ & $\begin{array}{c}\text { Normal } \\
\text { emotions }\end{array}$ & $\begin{array}{c}\text { Mild } \\
\text { symptoms }\end{array}$ & $\begin{array}{c}\text { Severe } \\
\text { symptoms }\end{array}$ \\
\hline \multirow{3}{*}{ Depression } & Normal & 0.937 & 0.014 & 0.000 & 0.923 & 0.001 & 0.000 \\
\hline & Mild-depression & 0.063 & 0.931 & 0.023 & 0.076 & 0.931 & 0.005 \\
\hline & Severe-depression & 0.000 & 0.055 & 0.977 & 0.001 & 0.068 & 0.995 \\
\hline \multirow{3}{*}{ Anxiety } & Normal & 0.993 & 0.017 & 0.000 & 0.977 & 0.000 & 0.000 \\
\hline & Mild-anxiety & 0.006 & 0.967 & 0.139 & 0.023 & 0.975 & 0.093 \\
\hline & Severe-anxiety & 0.001 & 0.015 & 0.861 & 0.001 & 0.025 & 0.907 \\
\hline
\end{tabular}

JLCPA $=$ joint latent class profile analysis; JLTA = joint latent transition analysis.

Table 6: The tertiary measurements estimates of $\gamma$ - and the profile prevalence $\delta$-parameters from JLCPA

\begin{tabular}{clccc}
\hline \hline Profile & \multicolumn{1}{c}{ Joint class } & Wave 1 & Wave 2 & Wave 3 \\
\hline \multirow{2}{*}{ Deterioration (71.6\%) } & Normal emotions & 0.621 & 0.541 & 0.477 \\
& Mild symptoms & 0.259 & 0.370 & 0.388 \\
& Severe symptoms & 0.120 & 0.089 & 0.135 \\
\hline \multirow{2}{*}{ Polarization (28.4\%) } & Normal emotions & 0.238 & 0.267 & 0.405 \\
& Mild symptoms & 0.518 & 0.502 & 0.291 \\
& Severe symptoms & 0.234 & 0.231 & 0.304 \\
\hline \hline
\end{tabular}

JLCPA = joint latent class profile analysis.

Table 7: The initial joint class prevalence estimates of $\delta$ - at wave 1 and transition probability $\tau$-parameter from JLTA

\begin{tabular}{lcccccccc}
\hline \hline & \multicolumn{3}{c}{ Joint class for Emotional well-being } \\
\cline { 2 - 5 } \multicolumn{1}{c}{ Joint Class } & \multicolumn{3}{c}{ Wave 2 } & & \multicolumn{3}{c}{ Wave3 } \\
\cline { 2 - 5 } & & $\begin{array}{c}\text { Normal } \\
\text { emotions }\end{array}$ & $\begin{array}{c}\text { Mild } \\
\text { symptoms }\end{array}$ & $\begin{array}{c}\text { Severe } \\
\text { symptoms }\end{array}$ & & $\begin{array}{c}\text { Normal } \\
\text { emotions }\end{array}$ & $\begin{array}{c}\text { Mild } \\
\text { symptoms }\end{array}$ & $\begin{array}{c}\text { Severe } \\
\text { symptoms }\end{array}$ \\
\hline Normal emotions (0.503) & 0.736 & 0.228 & 0.036 & & 0.698 & 0.271 & 0.032 \\
Mild symptoms (0.390) & Wave 1 & 0.334 & 0.559 & 0.107 & Wave 2 & 0.332 & 0.502 & 0.166 \\
Severe symptoms (0.107) & 0.176 & 0.275 & 0.549 & & 0.227 & 0.394 & 0.380 \\
\hline \hline
\end{tabular}

JLTA $=$ joint latent transition analysis.

1 tend to maintain at the same class Normal emotions with the probability of 0.736 , while some move to Mild symptoms with probability of 0.228 , and few move to Severe symptoms with probability of 0.036. That is, JLTA provides transition parameter estimates for two adjacent time points, such as the transition probability from wave 1 to wave 2 and the transition probability from wave 2 to wave 3. Transition probabilities are useful to explain the tendency to shift or maintain among a latent class for a given time point, in a pairwise way. However, these pairwise transition estimates are difficult to interpret for whole time period, because the number of possible combinations of joint latent classes significantly increases $\left(J^{T}\right)$ as time points $T$ increases. In contrast, JLCPA is useful for identifying how joint latent class prevalence change over time, and it does not need the combination of joint latent class. Therefore, JLCPA is easier to understand or interpret the flow over the entire period. Note that the more time points one considers, the more useful JLCPA would be versus JLTA.

\subsection{Estimates by adolescent-parent relationship group}

We now expand the discussion by considering the relationship with parents that affect the emotional well-being of adolescents, and perform our JLCPA according to the parent-adolescent-relationship. 
Table 8: The contribution of manifest items for subgrouping parent-adolescent relationship

\begin{tabular}{lccc}
\hline \hline \multirow{2}{*}{ Manifest item } & \multicolumn{3}{c}{ Class for Parent-adolescent relationship } \\
\cline { 2 - 4 } & Trust \& no anger & Trust \& squabbling & Apathy \\
\hline Seek parent's attention & 0.937 & 0.958 & 0.425 \\
Communication & 0.937 & 0.863 & 0.139 \\
Trust their parents & 0.985 & 0.975 & 0.624 \\
Not discussing problems w/ them & 0.782 & 0.348 & 0.530 \\
Rarely getting upset & 0.754 & 0.118 & 0.391 \\
Rarely avoiding parents & 0.697 & 0.362 & 0.439 \\
Rarely feeling angry & 0.812 & 0.462 & 0.337 \\
\hline \hline
\end{tabular}

Table 9: The estimated tertiary measurements according to the relationship with parent

\begin{tabular}{|c|c|c|c|c|c|}
\hline Relationship group & Profile & Joint class & Wave 1 & Wave 2 & Wave 3 \\
\hline \multirow{6}{*}{ Trust \& no anger } & \multirow{3}{*}{ Deterioration } & Normal emotions & 0.870 & 0.879 & 0.759 \\
\hline & & Mild symptoms & 0.108 & 0.121 & 0.241 \\
\hline & & Severe symptoms & 0.022 & 0.000 & 0.000 \\
\hline & \multirow{3}{*}{ Polarization } & Normal emotions & 0.382 & 0.073 & 0.123 \\
\hline & & Mild symptoms & 0.617 & 0.812 & 0.708 \\
\hline & & Severe symptoms & 0.001 & 0.115 & 0.169 \\
\hline \multirow{6}{*}{ Trust \& squabbling } & \multirow{3}{*}{ Deterioration } & Normal emotions & 0.450 & 0.571 & 0.584 \\
\hline & & Mild symptoms & 0.526 & 0.385 & 0.325 \\
\hline & & Severe symptoms & 0.024 & 0.044 & 0.091 \\
\hline & \multirow{3}{*}{ Polarization } & Normal emotions & 0.000 & 0.017 & 0.117 \\
\hline & & Mild symptoms & 0.510 & 0.458 & 0.483 \\
\hline & & Severe symptoms & 0.490 & 0.525 & 0.400 \\
\hline \multirow{6}{*}{ Apathy } & \multirow{3}{*}{ Deterioration } & Normal emotions & 0.440 & 0.467 & 0.382 \\
\hline & & Mild symptoms & 0.510 & 0.533 & 0.498 \\
\hline & & Severe symptoms & 0.050 & 0.000 & 0.120 \\
\hline & \multirow{3}{*}{ Polarization } & Normal emotions & 0.000 & 0.139 & 0.466 \\
\hline & & Mild symptoms & 0.092 & 0.115 & 0.000 \\
\hline & & Severe symptoms & 0.908 & 0.746 & 0.534 \\
\hline
\end{tabular}

For the parent-adolescent relationship, we divided sample youths into subgroups using 7 items at wave 1: whether do you (a) seek your caregiver's point of view; (b) tell your caregiver about problem; (c) trust your caregiver; (d) feel satisfied discussing problems with your caregiver; (e) rarely get upset about your caregiver; (f) never hesitate to bother your caregiver with problem; (g) rarely feel angry with your caregiver. Here, all the polychotomous items were also changed into dichotomous.

The optimal segmentation was determined as three groups by LCA in terms of model interpretation and simplicity ( $\mathrm{AIC}=5848.8, \mathrm{BIC}=5957.6$ ). The subgroups were labeled as 'Trust \& no anger', 'Trust \& squabbling', and 'Apathy', based on the estimated item-response probabilities as presented in Table 8. Table 9 shows that the sequential patterns of Adolescent emotional well-being differs among subgroups depending on the relationship with parent. Adolescents in 'Trust \& no anger' group having good relationship with their parents, have consistently high probabilities of belonging to the Normal emotion higher than 0.75 , implying that they are likely to remain normal over time, while their probabilities of Severe symptoms are almost zero over time. However, adolescents in the 'Trust \& squabbling' group show less tendency of belonging to Normal emotion than those in 'Trust \& no anger' group, and higher tendencies of having symptoms either Mild symptoms or Severe symptoms. For the 'Apathy' group, the joint class prevalence of Severe symptoms in Polarization profile shows the highest tendencies among the three relationship groups. These results imply that the negative relationship with parent may have an adverse effect on adolescent's emotional wellbeing. 


\section{Conclusion}

This paper proposes joint latent class analysis for longitudinal data in two different approaches of the as joint latent class profile analysis (JLCPA) and joint latent transition analysis (JLTA). By reflecting the dependencies among latent variables in cross-sectional and longitudinal view, our models allows an examination of the associations among latent variables of different attributes as well as an investigation of the joint stage-sequential progression for the mixture of multiple latent class variables. These models can be applied to other research subjects such as disease progression considering gene-gene interactions, or poly-substance use behavior patterns consisted of alcohol and cigarettes. We applied our models to investigate the sequential patterns of adolescents' emotional well-being, depression and anxiety, which are repeatedly measured as several survey items. Empirical results revealed three joint latent classes of normal emotions, mild symptoms, and severe symptoms, under both JLCPA and JLTA. JLCPA seems more useful to interpret than JLTA as time points to consider increase. Our JLCPA results presented two representative profiles for the adolescent emotional well-being over time, and their tendencies showed different according to the parent-adolescent-relationship subgroups. The proposed models can be generalized to incorporate covariates of individual characteristics, such as gender, race, and age. However, such inclusion of covariates may cause computational complexity requiring alternative estimation methods, which are left to future studies.

\section{Acknowledgements}

This work was supported by Basic Science Research Program through the National Research Foundation of Korea (NRF) by the Ministry of Education (2017R1C1B5077065 to Saebom Jeon and 2018R1D1A1B07045821 to Hwan Chung).

\section{References}

Angel R, Burton L, Chase-Lansdale LP, Cherlin A, and Moffitt R (2009). Welfare, Children, and Families: A Three-City Study, ICPSR04701-v7. Ann Arbor, MI: Inter-university Consortium for Political and Social Research.

Center for Behavioral Health Statistics and Quality (2018). Key substance use and mental health indicators in the United States: Results from the 2017 National Survey on Drug Use and Health, Substance Abuse and Mental Health Services Administration, Rockville, MD.

Collins LM and Lanza ST (2010). Latent Class and Latent Transition Analysis: With Applications in the Social, Behavioral, and Health Sciences, 718, John Wiley \& Sons.

Chung H, Anthony JC, and Schafer JL (2011). Latent class profile analysis: an application to stage sequential processes in early onset drinking behaviours, Journal of the Royal Statistical Society. Series A: Statistics in Society, 174, 689-712.

Chung H, Lanza ST, and Loken E (2008). Latent transition analysis: inference and estimation, Statistics in Medicine, 27, 1834-1854.

Garber J and Weersing VR (2010). Comorbidity of anxiety and depression in youth: Implications for treatment and prevention, Clinical Psychology: Science and Practice, 17, 293-306.

Gutman LM, McLoyd VC, and Tokoyawa T (2005). Financial strain, neighborhood stress, parenting behaviors, and adolescent adjustment in urban African American families, Journal of Research on Adolescence, 15, 425-449.

Horn PJ and Wuyek LA (2010). Anxiety disorders as a risk factor for subsequent depression, International Journal of Psychiatry in Clinical Practice, 14, 244-247. 
Jeon S, Lee J, Anthony JC, and Chung H (2017). Latent class analysis for multiple discrete latent variables: a study on the association between violent behavior and drug-using behaviors, Structural Equation Modeling: A Multidisciplinary Journal, 24, 911-925.

Kreuter F, Yan T, and Tourangeau R (2008). Good item or bad - can latent class analysis tell?: the utility of latent class analysis for the evaluation of survey questions Journal of the Royal Statistical Society: Series A (Statistics in Society), 171, 723-738.

Lee JW and Chung H (2017). Latent class analysis with multiple latent group variables, Communications for Statistical Applications and Methods, 24, 173-191.

Mooijaart AB and Van der Heijden PG (1992). The EM algorithm for latent class analysis with equality constraints, Psychometrika, 57, 261-269.

Nurius PS and Macy RJ (2008). Heterogeneity among violence-exposed women: applying personoriented research methods, Journal of Interpersonal Violence, 23, 389-415.

Schleider JL, Krause ED, and Gillham JE (2014). Sequential comorbidity of anxiety and depression in youth: present knowledge and future directions, Current Psychiatry Reviews, 10, 75-87.

van Lang ND, Ferdinand RF, Ormel J, and Verhulst FC (2006). Latent class analysis of anxiety and depressive symptoms of the youth self-report in a general population sample of young adolescents, Behaviour Research and Therapy, 44, 849-860.

White RMB and Roosa MW (2012). Neighborhood contexts, fathers, and Mexican American young adolescents' internalizing symptoms, Journal of Marriage and Family, 74, 152-166. 